\title{
Design and Implementation of Network Devices Monitoring System Based on SNMP
}

\author{
Liang Liu ${ }^{1}$ \\ College of Mobile Telecommunication \\ Chongqing University of Posts and \\ Telecommunications \\ Chongqing China \\ 241306755@qq.com
}

\author{
Weifeng Wang ${ }^{2}$ \\ College of Mobile Telecommunication \\ Chongqing University of Posts and \\ Telecommunications \\ Chongqing China \\ 21973433@qq.com
}

\author{
Zhilei Wang $^{3}$ \\ College of Computer Science and \\ Technology \\ Chongqing University of Posts and \\ Telecommunications \\ Chongqing, China \\ huajin26@163.com
}

\begin{abstract}
People requirement network reliability and security more and higher as the information age of the Internet to promote the rapid development of business and Internet using unceasingly thorough, network equipment, network structure is complicated. This paper design and implementation of a monitoring system to monitor the work status of the network equipment, visual display of various indicators of the network, in the equipment after failure to timely, automatically to the network administrator alarm.
\end{abstract}

\section{Keywords- SNMP; networking equipment; monitoring system}

\section{INTRODUCTION}

With the rapid development of computer network technology, the internal network of the large and mediumsized enterprises have gradually built, how to effectively monitor and manage network devices is a problem. Network equipment, server and application performance status will directly affect the quality of the business. Network device or server failure unable to provide normal service allows businesses to enable enterprises to suffer. Therefore, it is necessary to design a network devices monitoring system, which can real-time monitoring equipment status. It also provides network administrators with a convenient way to view the status information of the network equipment to ensure normal and stable service.

\section{SNMP ARCHITECTURE}

\section{A. SNMP structure model}

Simple Network Management Protocol (SNMP)[1][2] architecture is divided into three parts: managed devices, SNMP manager and SNMP agent. The SNMP architecture is like as Figure 1.

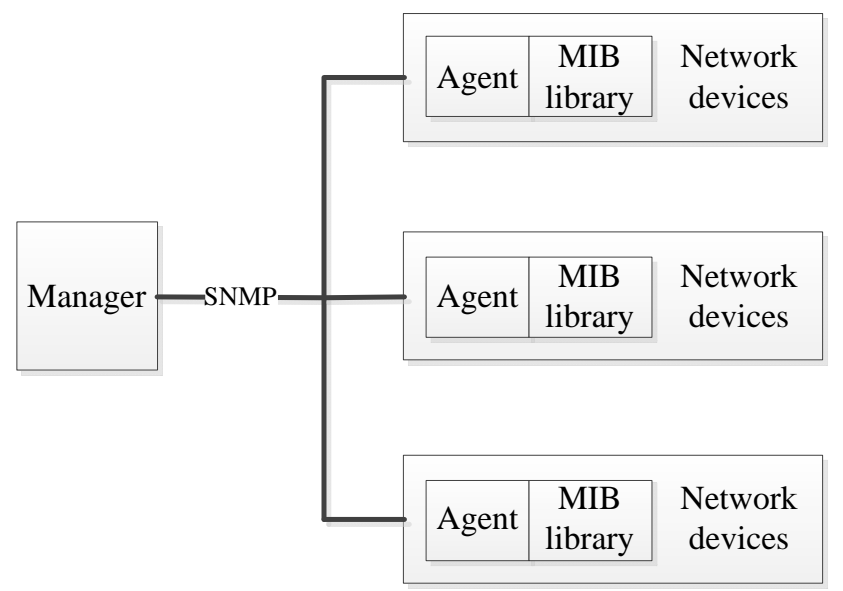

Figure 1. SNMP architecture diagram

The managed device is one of the network nodes, sometime called network element, it can be router, switch and other equipment support SNMP protocol.

SNMP manager manages network devices through the network management software; one of the main functions of the network management software is to help network administrators managing network devices. Network management software requires SNMP manager regularly collect important information of the device, the information collected will be used to determine the independent network equipment, part of the network or the entire network to run the state is normal. The SNMP manager regularly inquires the relevant equipment running status, configuration and performance of information which collected by the network devices' agent.

The SNMP Agent is a network management software module that resides on a managed device; it collects local device management information and translates that information into the form compatible with SNMP protocol.

\section{B. SNMP MIB}

The way of managing network resources is the resources to object to represent, each object represents managed the properties of a particular aspect of the resource, the collection of these objects form the Management Information Base (MIB)[3]. Management station complete monitoring and control function through reading and set the value of the 
MIB objects. The backbone devices agents are maintaining a MIB to reflect on the node of managed resources status. Network management entity can be monitor this node resource by reading object value in the MIB, and can be controlled these resources by modifying the value of the object.

\section{SNMP polling algorithm}

SNMP collect network devices data information by using self-trapping polling[4] method, generally, the networks management workstation collects data information by sending the GetRequest and GetNextRequest packets polling managed devices’ agent, Agents will response GetResponse message, this information display on the console through numeric or graphical , provide the working status of network devices, networks traffic analysis and management capabilities. When managed devices appear abnormal state, the management agent can also produce Trap message to administrators reporting the major changes of MIB and other important events. When a network device generates a selftrapped, the networks administrator can use the networks management station to query the status of the device, in order to get more information. Packets transfer relationship between manager and agent as shown in Figure 2.

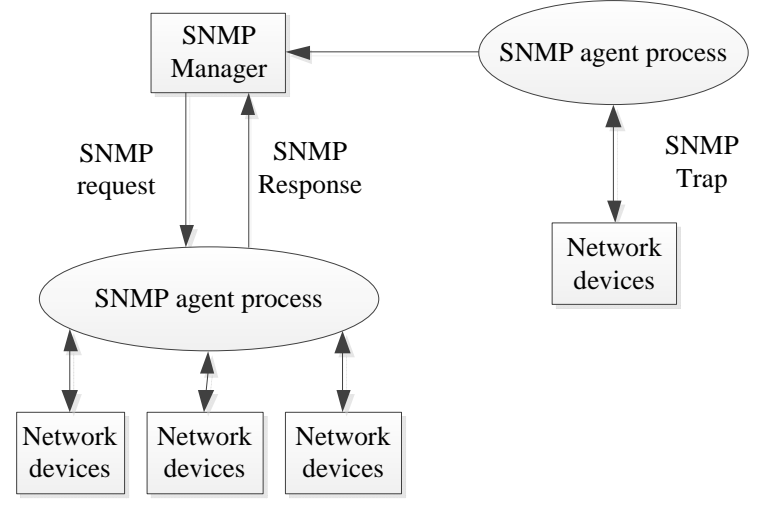

Figure 2. Packets transfer relationship between manager and agent

\section{SYSTEM DESIGN}

\section{A. System architecture}

Based on SNMP network monitor system design of the main goal is to realize the network topology management, network performance management[5], network event management, able to graphic form show each management operation interface, user-friendly, etc. The system consists of data collection layer, data processing layer and data application layer. The first layer is the data collection layer, which uses the SNMP protocol to communicate with the managed devices and completes of the collection of MIB information, ensure the integrity of the data collection[6]; the second layer is the data processing layer, which completes data calculation, storage, arrangement and organization; the third layer is the data the application layer, graphical interface, facing the administrator, the network topology display, network performance analysis results display, alarm events management operation and network event management and display. From the point of view of the longitudinal division, the function of the system module can be divided into two systems:

a) Networks data communication system, it is the basis and core of this system which solves the SNMP protocol, UDP protocol, IP protocol communication problem. The first layer and the second is the network data communication subsystem.

b) User interface system, The user interface system, which provides a friendly interface form for the user to use and provides all functions of the application by visible operational Chinese form of operator interface . The third layer is the user interface system. The overall system design is shown in Figure 3

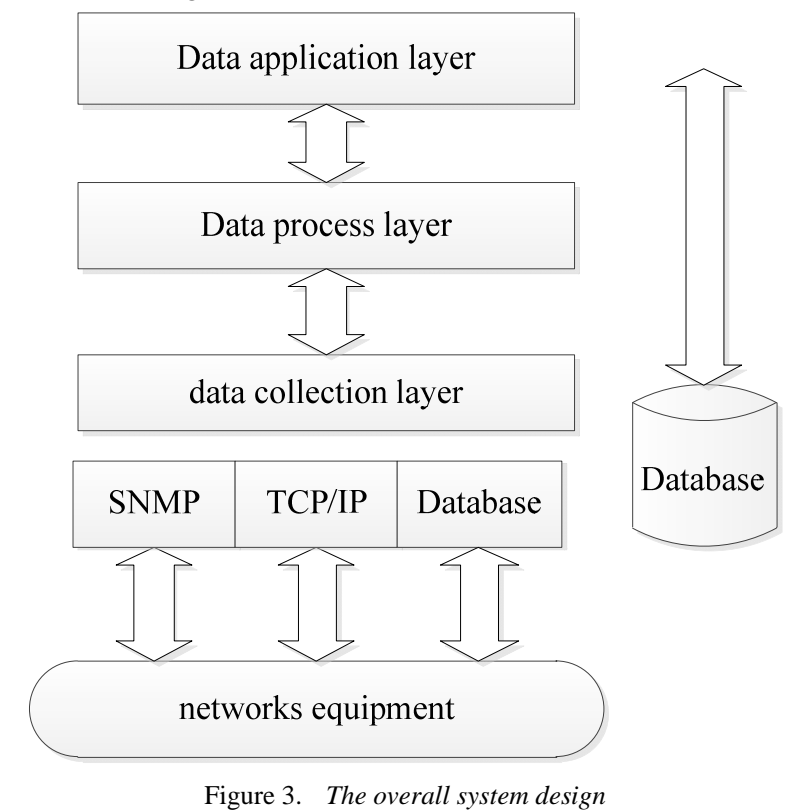

There are two main structural model of distributed systems: client/server (referred to as the C/S) model and browser/server (referred to as the $\mathrm{B} / \mathrm{S}$ ) model. The $\mathrm{C} / \mathrm{S}$ model with higher security requirements, communication ability, relatively fixed location, small computer distribution; The $\mathrm{B} / \mathrm{S}$ model is suitable for use range is wider, locations flexible, frequent changes in function, but safety and interactivity less demanding conditions. In paper proposes the system design, combines them with each other, its application conditions to determine the various parts of the system based on $\mathrm{C} / \mathrm{S}$ model or the $\mathrm{B} / \mathrm{S}$ model. The network data communication system executes SNMP protocol by the $\mathrm{C} / \mathrm{S}$ module from the network device in real time read the required data and stores the read data into the database; the user interface system adopts the B/S structure, the browser proposes data requirements to the Web Server according to the various requirements of the use.

\section{B. System functional description}

a) Collect performance data on the managed devices 
Collecting data about real-time status of managed devices on the network monitoring system is crucial. Through the server collects the network devices data can be real-time monitoring to network equipment state information. The use of data collection module can realtime collect data, display performance status of managed devices , also collect information on time and save it, the formation of historical data About the performance of the managed devices.

b) Performance information analysis processing

For real time data, taking corresponding calculation formula analysis according to the corresponding data to determine the utilization[7] of the network, etc. For historical data, professional analysis tools can be used for network trend analysis, historical data network operation status to predict peak network usage, thus avoiding network saturation may bring low performance.

\section{c) Alarm function}

Before the user attention to abnormal performance issued a warning, can active preventive found network and the application may appear problem places to help prevent performance down.

The template is used to format your paper and style the text. All margins, column widths, line spaces, and text fonts are prescribed; please do not alter them. You may note peculiarities. For example, the head margin in this template measures proportionately more than is customary. This measurement and others are deliberate, using specifications that anticipate your paper as one part of the entire proceedings, and not as an independent document. Please do not revise any of the current designations.

\section{Network data communication system design}

The design of the network data communication system consists of two parts: data collection and data analysis.

The data collection module is a critical part of the whole network device monitoring system; it is the first part of the network device monitoring process and the foundation of business processing, data storage and other follow-up links. No data collection, network device monitoring system will lose the sources of information and follow-up of sectors of the processing elements are lost, there is no function for the entire system. The importance of the data collected, the decision must be taken into account in the design of the module to achieve high real-time, high reliability, so that the accuracy of data collection, real-time, high-key factors affect the performance monitoring system performance.

The data analysis[8] is a new collection of data for timely analysis and compare network performance thresholds set in the system, if the threshold is exceeded, the police immediately.

\section{The user interface system design}

The design of the client browser-based, in order to ensure that only authorized users can view the device information, the browser side program provides the functionality to authenticate users. Users must to authenticate before using the system, when through certification, they can see list of equipment within the scope of authorization. Each entry in the list is a hyperlink, to get the query results when the user clicks on a link, the browser-side program will interact with the server-side program. The design of the difficulty lies in how to implement the program of browser-side communication with the server-side program, we establish Socket between them for communication. Since both programs are installed on the same computer, so we use the IP address of the loopback address (127.0.0.1) as Socket connection. Using the loopback address communication, data is not transmitted on the network; it can guarantee the security of communication. In addition, both programs also need to specify a common port as the Socket connection port can be any one of the free high-end port (port number 1024) port.

\section{SYSTEM IMPLEMENTATION}

In this paper, using PHP language developed the network equipment monitoring system based on SNMP, and has been run in a real environment, this system is deployed in a large IP network, capable of real-time monitoring of the entire network device status information.

A network device data collection run results is like as Figure 4.

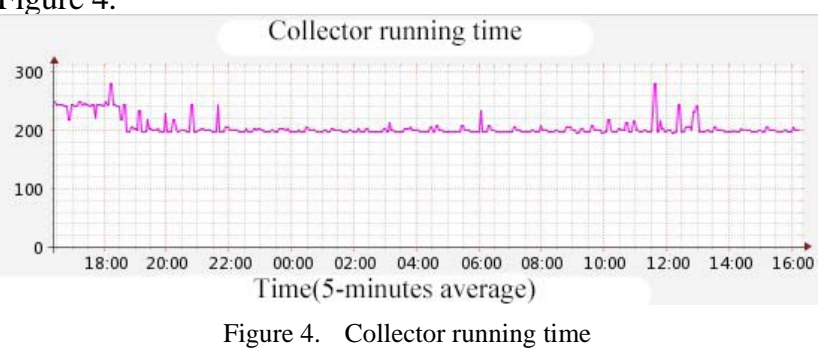

The CPU utilization is depicted in Figure 5 that the network devices monitoring system gathers a device CUP usage each period of the day.

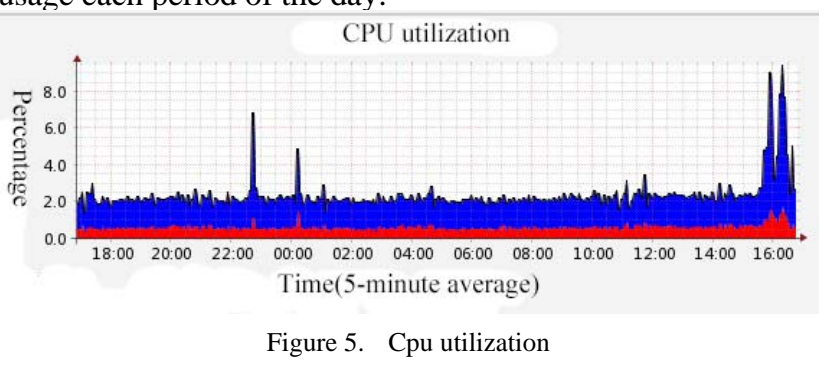

Network monitoring system also can get the memory utilization of the network devices, the device network traffic and the load average information of the device, respectively as shown in Figure. 6, 7, 8. 


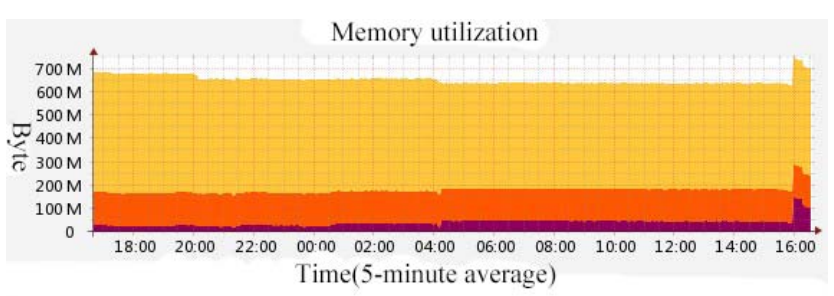

Figure 6. Memory utilization

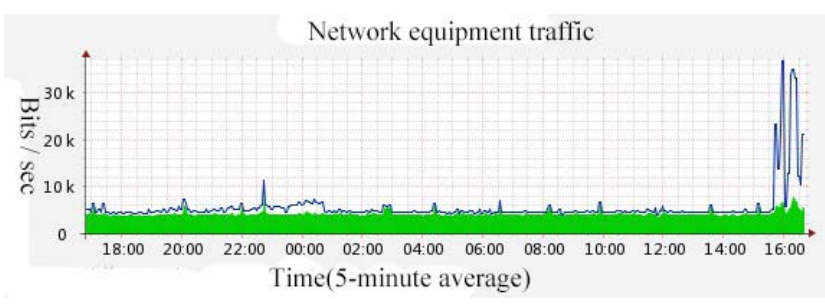

Figure 7. Network equipment traffic

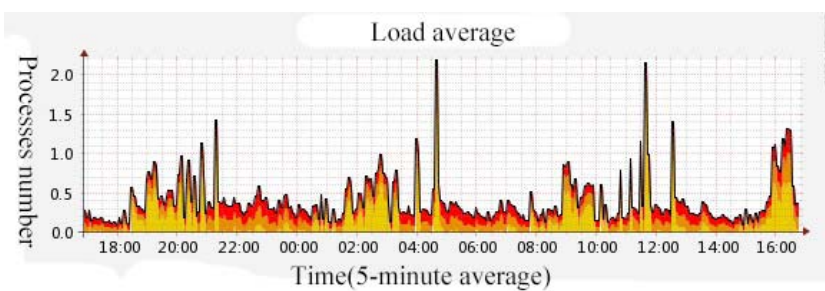

Figure 8. Load average

\section{CONCLUSION}

This paper discusses the architecture based SNMP network monitoring system, the system successfully achieved most of the basic functions such as SNMP information collection module, data analysis module and so on. And through the Web server status information real-time display, fault real-time warning, graphical reports display, custom functions.

\section{ACKNOWLEDGMENT}

This work is supported by the Department of Computer Science, College Mobile Telecommunications Chongqing University of Posts and Telecom.

\section{REFERENCES}

[1] Rose M. RFC 1213-1991, Management information base for network management of TCP/IP-based internets: MIB - I I[S]

[2] 13]Case J, Fedor M, Schoffstall M, et a1. The Simple Network Management Protocol(SNMP)[S]. RFC1 157.1990.

[3] K. McCloghrie, M. Rose. Management Information Base for Network Management of TCP/IP. based internets: MIB. II RFC 1213. 1991. 1-13.

[4] K. Ohta,N. Sun,G,Mans. Eld,and Y.Nemeto:Elective polling control for network management using SNMP,IEICE,Technical Reports on Informarion Network.1N94-135 : 91 96,Nov,1994.

[5] P Phaal, S Panchen, N McKee. InMon Corporation'S sFlow: A Method for Monitoring Trafic in Switched and Routed Networks[Z], RFC 3176, 2001.

[6] Harrington D, Presuhn R, Wijnen B. An Architecture for Describing SNMP Managemnet Frameworks[S]. RFC1157, 1999.

[7] IETF. rfcl905. Protocol Operations for Version 2 of the Simple Network Management Protocol(SNMPv2). SNMPv2 Working Group, J. Case, K. McCloghrie, M. Rose, S. Waldbusser, 1996-l.

[8] A. Correnm, L. Tufa.Security performance analysis of SNMPv3 with respect to SNMPv2c. Network Operations and Management Symposium. IEEEflFIE2004. 1.729—742. 